\title{
Random ballistic interpretation of the nonlinear guiding center theory of perpendicular transport
}

\section{Ruffolo ${ }^{* a}$ T. Jitsuk, ${ }^{a}$ T. Pianpanit,${ }^{a}$ A. P. Snodin,,${ }^{b}$ W. H. Matthaeus, ${ }^{c}$ P. Chuychai ${ }^{d}$}

${ }^{a}$ Mahidol University, Bangkok, Thailand. ${ }^{b}$ King Mongkut's University of Technology North

Bangkok, Bangkok, Thailand. ${ }^{c}$ University of Delaware, Newark, USA. ${ }^{d}$ Mae Fah Luang

University, Chiang Rai, Thailand.

E-mail: david.ruf@mahidol.ac.th, taweesak.jit@student.mahidol.ac.th,

th_ee@hotmail.com, andrew.snodin@gmail.com, whm@udel.edu,

piyanate@gmail.com

\begin{abstract}
Nonlinear guiding center (NLGC) theory has been used to explain the asymptotic perpendicular diffusion coefficient $\kappa_{\perp}$ of energetic charged particles in a turbulent magnetic field, which can be applied to better understand cosmic ray transport. Here we re-derive NLGC, replacing the assumption of diffusive decorrelation with random ballistic decorrelation (RBD), which yields an explicit formula for $\kappa_{\perp}$. We note that scattering processes can cause a reversal of the guiding center motion along the field line, i.e., "backtracking," leading to partial cancellation of contributions to $\kappa_{\perp}$, especially for low-wavenumber components of the magnetic turbulence. We therefore include a heuristic backtracking correction (BC) that can be used in combination with RBD. In comparison with computer simulation results for various cases and two different magnetic field models (2D+slab and noisy RMHD), NLGC with RBD and BC provides a substantially improved characterization of the perpendicular diffusion coefficient for a fluctuation amplitude less than or equal to the large-scale magnetic field.
\end{abstract}

The 34th International Cosmic Ray Conference,

30 July- 6 August, 2015

The Hague, The Netherlands

\footnotetext{
* Speaker.
} 


\section{Introduction}

While charged particles subject to a magnetic field in a tenuous plasma will mainly gyrate along that field, magnetic turbulence can cause particles to also spread in the directions perpendicular to the large-scale field. Such perpendicular transport involves an interesting interplay between the transport along field lines, the random walk of magnetic field lines perpendicular to the largescale field direction, and true cross-field transport in which the particle guiding center eventually separates from its original field line.

The classic FLRW theory [11], in which particles follow magnetic field lines with a fixed pitch angle, directly related the perpendicular diffusion coefficient $\kappa_{\perp}$ to the field line diffusion coefficient $D$. Meanwhile another viewpoint in terms of scattering led to a relation between $\kappa_{\perp}$ and the parallel diffusion coefficient $\kappa_{\|}[1,10]$. Nonlinear guiding center (NLGC) theory [14] successfully accounts for both factors, allowing the guiding center motion to decorrelate due to both parallel (pitch angle) scattering and the random walk of the guiding magnetic field line, for transverse magnetic fluctuations with a general power spectrum. This theory has provided a much closer match to observations [3] and computer simulation results for $\kappa_{\perp}$ (see also [15, 19]), and its framework has attracted theoretical interest and inspired numerous related theories, e.g., [12, 17, $18,25,27,28]$.

In the present work, we consider an alternate interpretation of NLGC that replaces the diffusive decorrelation (DD) of guiding center trajectories with random ballistic decorrelation (RBD), for the purpose of calculating the Lagrangian magnetic correlation function. This approach was introduced for calculating the field line diffusion coefficient and led to some substantial improvements in the match with direct simulation results [9]. It is analogous to concepts in random walk theory in which the mean free path is determined by the extent of ballistic motion between scattering events. We consider that decorrelation of the particle velocity takes place over a distance scale for which the parallel motion is approximately constant and the field lines are approximately straight, so the guiding center motion can be treated as ballistic in random directions determined by the distribution of magnetic field directions (Figure 1). To test this version of the theory, we have previously performed computer simulations of particle trajectories in the 2D+slab model of magnetic turbulence [22], and in this work we also report simulations in the noisy RMHD model as defined by [20]. We demonstrate that the RBD approach, together with a backtracking correction, leads to a substantial improvement in the match with direct computer simulations of the perpendicular diffusion of energetic charged particles.

\section{Random ballistic interpretation of nonlinear guiding center theory}

Here we outline the random ballistic interpretation of NLGC theory, which was more derived in more detail by [22]. We consider the diffusion of particles perpendicular to a mean field $B_{0} \hat{\mathbf{z}}$, subject to transverse, axisymmetric fluctuations $b_{x}$ and $b_{y}$. As in the original derivation of NLGC, we use

$$
\kappa_{x x}=\frac{a^{2} v^{2}}{3 B_{0}^{2}} \int_{0}^{\infty} e^{-t / \tau}\left\langle b_{x}(0,0) b_{x}[\mathbf{x}(t), t]\right\rangle d t
$$




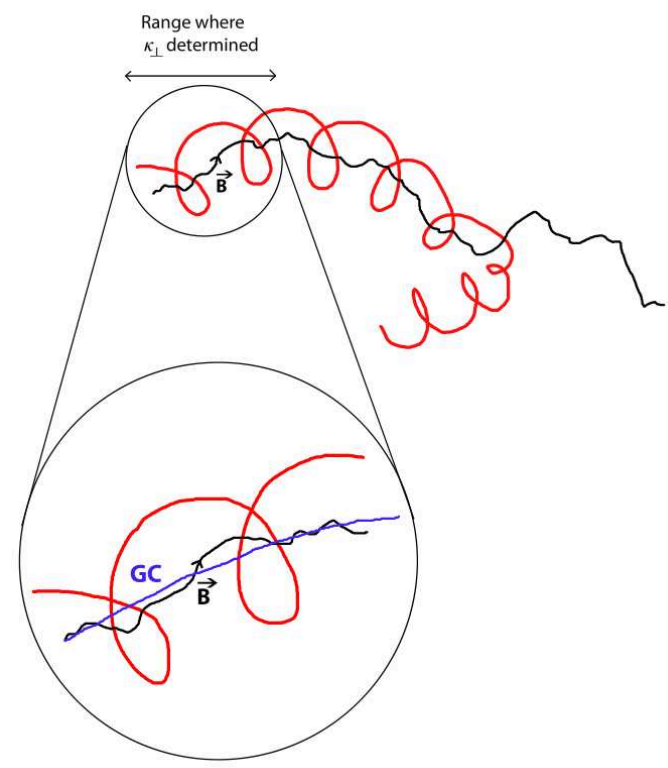

Figure 1: Illustration of the random ballistic interpretation of nonlinear guiding center (NLGC) theory. The diffusion coefficient $\kappa_{\perp}$ of energetic charged particle motion (red line) perpendicular to the largescale magnetic field is related to the decorrelation (i.e., change in direction) of a perpendicular component of the guiding center velocity (GC; blue line), which roughly follows a local magnetic field line (black line). Over the relevant distance scale, the guiding center motion can be approximated as ballistic (i.e., with constant velocity) along random directions distributed like the magnetic field directions. Such random ballistic decorrelation (RBD) is determined using the framework of NLGC theory, including the effects of the field line random walk and the parallel scattering of particle trajectories.

where $\kappa_{x x}$ is the particle diffusion coefficient in the perpendicular direction $x, a^{2}=1 / 3, v$ is the particle speed, and $\tau$ is the mean free time of parallel (pitch-angle) scattering. We then make use of Corrsin's independence hypothesis [7] to relate the Lagrangian correlation $\left\langle b_{x}(0,0) b_{x}[\mathbf{x}(t), t]\right\rangle$ to the Eulerian correlation function $R_{x x}$ and the probability of displacement $\mathbf{x}$ at time $t$, so that

$$
\kappa_{x x}=\frac{a^{2} v^{2}}{3 B_{0}^{2}} \int_{0}^{\infty} e^{-t / \tau} \int R_{x x}(\mathbf{x}, t) P(\mathbf{x} \mid t) d \mathbf{x} d t .
$$

Following [14], we use the Fourier transform of the correlation function $R_{x x}(\mathbf{x}, t)$ as the power spectrum $S_{x x}(\mathbf{k}, t)=S_{x x}(\mathbf{k}) e^{-\gamma(\mathbf{k}) t}$ and assume independent, Gaussian guiding center displacement probability distributions. For RBD we use a variance $\sigma_{i}^{2}=\left\langle\tilde{v}_{i}^{2}\right\rangle t^{2}$, for mean square guiding center velocity components as follows:

$$
\begin{gathered}
\left\langle\tilde{v}_{x}^{2}\right\rangle=\left\langle\tilde{v}_{y}^{2}\right\rangle \approx \frac{a^{2}}{B_{0}^{2}}\left\langle v_{z}^{2}\right\rangle\left\langle b_{x}^{2}\right\rangle=\frac{a^{2} v^{2}}{6} \frac{b^{2}}{B_{0}^{2}} \\
\left\langle\tilde{v}_{z}^{2}\right\rangle=\frac{v^{2}}{3}\left(1-a^{2} \frac{b^{2}}{B_{0}^{2}}\right) .
\end{gathered}
$$

This yields

$$
\kappa_{x x}=\frac{a^{2} v^{2}}{3 B_{0}^{2}} \int S_{x x}(\mathbf{k}) T(\mathbf{k}) d \mathbf{k},
$$

where the mean free time $T(\mathbf{k})$ is given by

$$
T(\mathbf{k})=\int_{0}^{\infty} \exp \left[-\frac{t}{\tau}-\gamma(\mathbf{k}) t-\frac{1}{2} \sum_{i} k_{i}^{2}\left\langle\tilde{v}_{i}^{2}\right\rangle t^{2}\right] d t
$$

Performing the $t$-integration and using $1 / \tau=v / \lambda_{\|}=v^{2} /\left(3 \kappa_{z z}\right)$, we obtain

$$
T(\mathbf{k})=\sqrt{\frac{\pi}{2}} \frac{e^{\alpha^{2}} \operatorname{erfc}(\alpha)}{\sqrt{\sum_{i} k_{i}^{2}\left\langle\tilde{v}_{i}^{2}\right\rangle}}
$$


and

$$
\kappa_{x x}=\frac{a^{2} v^{2}}{3 B_{0}^{2}} \sqrt{\frac{\pi}{2}} \int \frac{S_{x x}(\mathbf{k})}{\sqrt{\sum_{i} k_{i}^{2}\left\langle\tilde{v}_{i}^{2}\right\rangle}} e^{\alpha^{2}} \operatorname{erfc}(\alpha) d \mathbf{k},
$$

where

$$
\alpha \equiv \frac{v^{2} /\left(3 \kappa_{z z}\right)+\gamma(\mathbf{k})}{\sqrt{2 \sum_{i} k_{i}^{2}\left\langle\tilde{v}_{i}^{2}\right\rangle}} .
$$

Note that the original DD interpretation of [14] used the formula for asymptotic diffusion with $\kappa_{x x}$ in the displacement distribution, yielding an implicit equation for $\kappa_{x x}$. In contrast, the RBD theory uses a predetermined random ballistic formula for the displacement distribution and yields an explicit formula for $\kappa_{x x}$, as in analogous theories for the field line diffusion coefficient [9]. For numerical evaluation, NLGC-type theories based on DD typically require an iterative solution, whereas NLGC/RBD can be evaluated without iteration.

As explained by [22], backtracking played a major role in the original NLGC/DD theory [14], with diffusive decorrelation (DD), but is not present in the RBD calculation, which is based on ballistic guiding center trajectories.

Therefore, we introduce a heuristic backtracking correction (BC) for RBD, multiplying $T(\mathbf{k})$ by $e^{-\alpha^{2}}$, which simplifies Equation (2.7) to yield

$$
\kappa_{x x}=\frac{a^{2} v^{2}}{3 B_{0}^{2}} \sqrt{\frac{\pi}{2}} \int \frac{S_{x x}(\mathbf{k})}{\sqrt{\sum_{i} k_{i}^{2}\left\langle\tilde{v}_{i}^{2}\right\rangle}} \operatorname{erfc}\left[\frac{v^{2} /\left(3 \kappa_{z z}\right)+\gamma(\mathbf{k})}{\sqrt{2 \sum_{i} k_{i}^{2}\left\langle\tilde{v}_{i}^{2}\right\rangle}}\right] d \mathbf{k} . \quad(\mathrm{RBD} / \mathrm{BC})
$$

Note also that in Eq. (2.6), for a given $\mathbf{k}$, there is a time $t$ when the linear and quadratic terms are equal, i.e., the field line random walk becomes important. At that time we have $t / \tau \sim \alpha^{2}$, and substitution into the parallel velocity correlation term $e^{-t / \tau}$ suggests the use of $e^{-\alpha^{2}}$ to account for backtracking effects.

We evaluate this theory of perpendicular diffusion for two models of transverse magnetic turbulence: the 2D+slab model and the noisy RMHD model. In the 2D+slab model, the power spectrum is a sum of a two-dimensional (2D) power spectrum, depending only on $k_{x}$ and $k_{y}$, and a slab power spectrum depending only on $k_{z}$. The latter represents parallel Alfvénic fluctuations and the former idealizes the quasi-2D structures, including "flux tubes," that can develop from interactions of such waves; see $[6,23,30]$ and references therein. The two-component model was motivated by observations of interplanetary magnetic fluctuations, indicating quasi-slab and quasi-2D components [13,31], which can be modeled using a ratio of slab:2D fluctuation energies of approximately 20:80 [4, 5]. This model has provided a useful description of the parallel transport of particles in the inner heliosphere [4], and was used by most studies that implemented or tested NLGC theory.

For the case of 2D+slab fluctuations, the expression for $\kappa_{\perp}$ splits into two terms using $S_{x x}^{\text {slab }}$ and $S_{x x}^{2 D}$. However, [24] has proposed that the direct contribution of slab fluctuations to the perpendicular transport should be subdiffusive, and that the $S_{x x}^{\text {slab }}$ term should not be included in the equation of $\kappa_{\perp}$. (Note that slab fluctuations can still play a role as a key determinant of $\lambda_{\|}$and $\kappa_{z z}$, which enters into the 2D contribution.) We refer to this proposal as the Shalchi slab hypothesis (SSH). We employ this in the present work, and for Equation (2.10) we use only the $S_{x x}^{2 D}$ term. Because $\nabla \times \mathbf{b}^{2 D} \equiv 0$, the components $b_{x}^{2 D}$ and $b_{y}^{2 D}$ are related through a potential function $a(x, y)$ 
by $\mathbf{b}^{2 \mathrm{D}}=\nabla \times[a(x, y) \hat{\mathbf{z}}]$, which implies that $S_{x x}\left(k_{x}, k_{y}\right)=k_{y}^{2} A\left(k_{\perp}\right)$ and $S_{y y}\left(k_{x}, k_{y}\right)=k_{x}^{2} A\left(k_{\perp}\right)$, where $A$ is the power spectrum of $a(x, y)$, which by axisymmetry is a function only of $k_{\perp} \equiv \sqrt{k_{x}^{2}+k_{y}^{2}}$.

The other model we consider, noisy reduced magnetohydrodynamics (noisy RMHD), is a synthetic model introduced by [20] in which the power spectrum is specified by an analytic function in order to approximate the form of transverse RMHD fluctuations, as generalized to allow a general Kubo number, $R=\left(b / B_{0}\right)\left(\ell_{c} / \ell_{\perp}\right)$, where $b$ is the rms magnetic fluctuation, and $\ell_{c}$ and $\ell_{\perp}$ are correlation scales parallel and perpendicular to the mean field, respectively. Now the statistically homogeneous fluctuating field is given by

$$
\mathbf{b}(x, y, z)=\nabla_{\perp} \times[a(x, y, z) \hat{\mathbf{z}}],
$$

where the subscript " $\perp$ " indicates a projection perpendicular to the mean field in which only $x$ - and $y$-components are included. In terms of wave vectors $\mathbf{k}$, we can write

$$
\mathbf{b}(\mathbf{k})=i \mathbf{k}_{\perp} \times[a(\mathbf{k}) \hat{\mathbf{z}}],
$$

and we specify the potential function in $\mathbf{k}$-space by

$$
a(\mathbf{k}) \propto\left\{\begin{array}{cl}
a^{2 \mathrm{D}}\left(k_{\perp}\right) e^{i \varphi(\mathbf{k})} & \text { for }\left|k_{z}\right| \leq K \\
0 & \text { for }\left|k_{z}\right|>K,
\end{array}\right.
$$

where $\varphi(\mathbf{k})$ is a random phase and $K=\pi /\left(2 \ell_{c}\right)$. In terms of power spectra this model gives [21]

$$
\begin{aligned}
& S_{x x}(\mathbf{k})=\left\{\begin{array}{cc}
k_{y}^{2} A\left(k_{\perp}\right) /(2 K) & \text { for }\left|k_{z}\right| \leq K \\
0 & \text { for }\left|k_{z}\right|>K
\end{array}\right. \\
& S_{y y}(\mathbf{k})=\left\{\begin{array}{cc}
k_{x}^{2} A\left(k_{\perp}\right) /(2 K) & \text { for }\left|k_{z}\right| \leq K \\
0 & \text { for }\left|k_{z}\right|>K,
\end{array}\right.
\end{aligned}
$$

where $A$ is the $2 \mathrm{D}$ power spectrum of $a^{2 \mathrm{D}}$. This "boxcar" dependence on $k_{z}$ has previously been used to characterize the results of RMHD simulations [16]. For both models we set $A\left(k_{\perp}\right)$ to yield Kolmogorov scaling at high $k_{\perp}$.

\section{Comparison between theory and simulation results}

We have also performed direct computer simulations to trace particle orbits in 2D+slab and noisy RMHD turbulence. While the simulations inevitably involve some discretization and statistical errors, they do avoid key assumptions of the analytic work, and thus provide an independent check of their validity. We first generated the magnetic fluctuations on a regular grid in Fourier space, $\mathbf{b}(\mathbf{k})$, with an amplitude corresponding to the desired power spectrum and a random complex phase. Then inverse Fourier transforms were used to generate $\mathbf{b}(\mathbf{x})$. We used a version of the Streamline code [8] to perform the particle tracing subject to $\mathbf{B}=B_{0} \hat{\mathbf{z}}+\mathbf{b}(\mathbf{x})$.

The computer simulations for the $2 \mathrm{D}+$ slab field were performed using the methods, power spectra, and parameter values described by [19]. In particular, distances are in units of $\lambda=0.02$ $\mathrm{AU}$, the slab and 2D turbulence bendover scale, and velocities are in units of the speed of light 

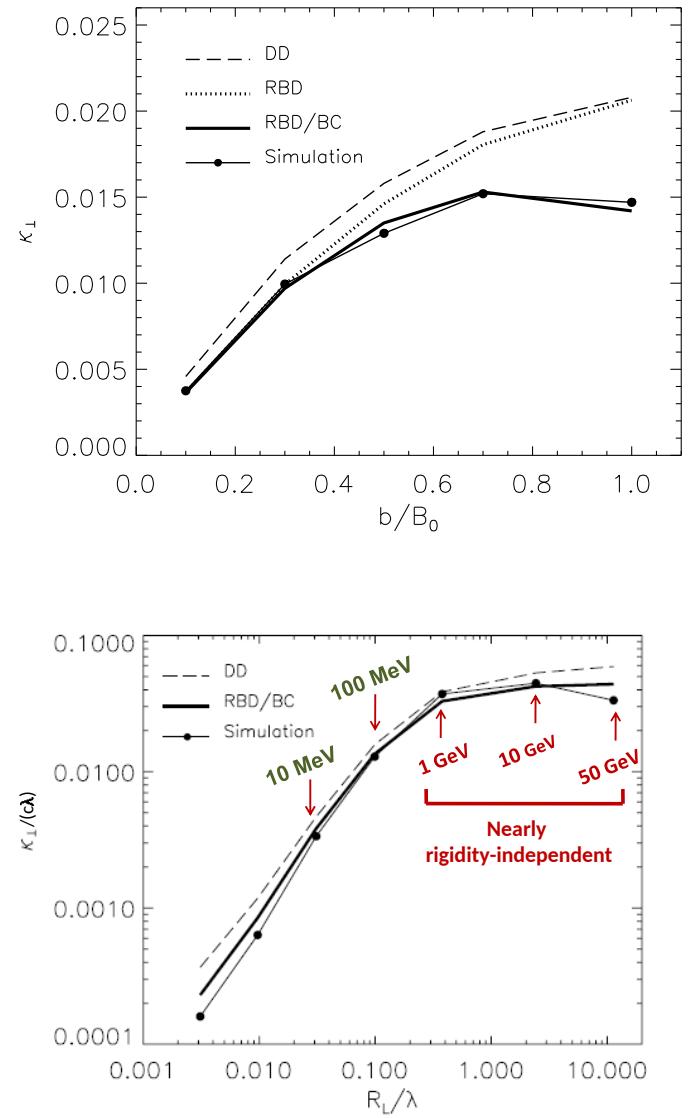

Figure 2: Asymptotic perpendicular diffusion coefficient $\kappa_{\perp}$ of $100 \mathrm{MeV}$ protons in 2D+slab turbulence with a slab fraction $f_{s}=0.2$ as a function of the magnetic fluctuation amplitude $b / B_{0}$. Using the NLGC framework, random ballistic decorrelation with backtracking correction ( $\mathrm{RBD} / \mathrm{BC}$; thick line) provides a closer match with computer simulation results (solid circles) than the original DD theory (long-dashed line) and uncorrected RBD (short-dashed line). In the present work we also employ the Shalchi slab hypothesis [24].

Figure 3: Asymptotic perpendicular diffusion coefficient $\kappa_{\perp}$ in 2D+slab turbulence with $f_{s}=0.2$ and $b / B_{0}=0.5$ as a function of the proton gyroradius in units of the turbulence bendover scale. The simulation values shown here (solid circles) correspond to proton energies ranging from $0.1 \mathrm{MeV}$ to $50 \mathrm{GeV}$ for $B_{0}=5 \mathrm{nT}$ and $\lambda=0.02 \mathrm{AU}$. In most cases, the $\mathrm{RBD} / \mathrm{BC}$ theory (thick line) provides a better explanation of the computer simulation results (solid circles) than the original DD theory (dashed line).

c. Simulations were performed over a sufficient time for all $\kappa_{i i}$ to approach asymptotic values, within statistical errors. We assume axisymmetry about the large-scale field direction, so $\kappa_{x x}$ and $\kappa_{y y}$ should be the same within statistical errors, which we verified in all cases. We report $\kappa_{\perp} \equiv$ $\left(\kappa_{x x}+\kappa_{y y}\right) / 2$, which can be compared directly with $\kappa_{x x}$ from theories.

Figure 2 shows the dependence of $\kappa_{\perp}$ (in units of $c \lambda$ ) on the overall fluctuation amplitude $b / B_{0}$, using $f_{s} \equiv b_{\text {slab }}^{2} /\left(b_{\text {slab }}^{2}+b_{2 \mathrm{D}}^{2}\right)=0.2$. It is apparent that the $\mathrm{RBD} / \mathrm{BC}$ version (thick lines) agrees with computer simulation results (solid circles) better and over a wider range of $b / B_{0}$ values than either the DD theory (long-dashed lines) or RBD without the backtracking correction (shortdashed lines), over the range of applicability of $\operatorname{RBD}\left(b / B_{0} \leq 1 / a=\sqrt{3}\right)$. We have also examined the dependence on the proton gyroradius (Figure 3), which is related to its energy, for fixed $f_{s}=0.2$ and $b / B_{0}=0.5$. The seven simulations were for protons of kinetic energy $0.1,1,10$, and $100 \mathrm{MeV}$ as well as 1,10 , and $50 \mathrm{GeV}$. The RBD results, not shown, nearly match DD at $R_{L} / \lambda<1$, nearly match $\mathrm{RBD} / \mathrm{BC}$ at $R_{L} / \lambda>1$, and are intermediate at $R_{L} / \lambda \approx 1$, Overall, the $\mathrm{RBD} / \mathrm{BC}$ theory again provides the best explanation of the computer simulation results.

We also show preliminary results for $\kappa_{\perp}$ vs. $b / B_{0}$ in noisy RMHD turbulence. Again, the $\mathrm{RBD} / \mathrm{BC}$ theory is in reasonable agreement with computer simulation results. Since this version of the theory was originally presented as being able to match computer simulation results for $2 \mathrm{D}+$ slab turbulence [22], it is particularly encouraging that the theory is also consistent with direct simulation results for another model of transverse turbulence. Note that perpendicular diffusion in noisy 


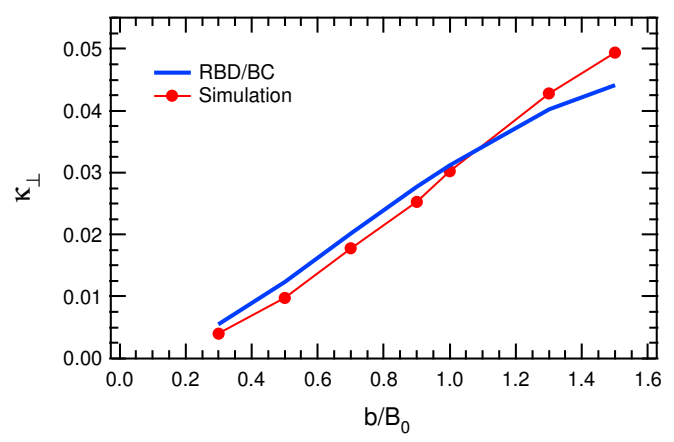

Figure 4: Asymptotic perpendicular diffusion coefficient $\kappa_{\perp}$ in noisy RMHD turbulence for charged particles of $v=0.3042 c$ with a Larmor radius $R_{L}$ equal to the parallel correlation length $\ell_{c}$ as a function of the magnetic fluctuation amplitude $b / B_{0}$.

RMHD turbulence has also been examined by [26, 29].

\section{Discussion}

Note that RBD theory does not require a small fluctuation amplitude, and indeed RBD/BC matches computer simulation results very well for amplitudes up to $b / B_{0} \sim 1$ (Figure 2). The inapplicability for $b / B_{0}>1 / a=\sqrt{3}$ [see Eq. (2.4)] indicates room for future improvements to obtain a truly non-perturbative theory. Note that NLGC itself assumes transverse fluctuations. In the interplanetary medium of the inner heliosphere, transverse fluctuations account for $\sim 90 \%$ of the magnetic fluctuation energy [2], so NLGC is well justified in this case.

The NLGC framework may be less appropriate when $R_{L} / \lambda \gg 1$, because it considers that guiding center motion tracks the local field line random walk, whereas low-wavelength fluctuations should have less influence on perpendicular diffusion when they are averaged over such a large gyroradius. We have searched for and found this effect at the highest proton energy shown in Figure $3,50 \mathrm{GeV}$, which corresponds to $R_{L} / \lambda=11$ for our parameter values of $B_{0}=5 \mathrm{nT}, \lambda \sim 0.02 \mathrm{AU}$, $b / B_{0}=0.5$, and $f_{s}=0.2$, which are reasonable for the interplanetary medium near Earth. The perpendicular diffusion coefficient $\kappa_{\perp}$ decreases, while all NLGC theories predict a slight increase. In any case, the above energies where $R_{L} \sim \lambda$ for interplanetary and interstellar propagation are sufficiently high that NLGC theories remain applicable to a wide range of cosmic ray and energetic particle transport problems.

\section{Acknowledgments}

This work was partially supported by the Thailand Research Fund, the Development and Promotion of Science and Technology Talents Project of the Royal Thai Government, the US NSF (AGS-1063439 and SHINE AGS-1156094), NASA Heliophysics Grand Challenge Research NNX14AI63G, the Solar Probe Plus/ISIS project, NASA LWS grant NNX15AB88G, and Thailand's Ministry of Education. We thank Achara Seripienlert for technical assistance.

\section{References}

[1] Axford, W. I. 1965, P\&SS, 13, 115

[2] Belcher, J. W., \& Davis, L., Jr. 1971, JGR, 76, 3534 
[3] Bieber, J. W., Matthaeus, W. H., Shalchi, A., \& Qin, G. 2004, GRL, 31, L10805

[4] Bieber, J. W., Matthaeus, W. H., Smith, C. W., Wanner, W., Kallenrode, M.-B., \& Wibberenz, G. 1994, ApJ, 420, 294

[5] Bieber, J. W., Wanner, W., \& Matthaeus, W. H. 1996, JGR, 101, 2511

[6] Borovsky, J. E. 2008, JGR, 113, A08110

[7] Corrsin, S. 1959, in Atmospheric Diffusion and Air Pollution, ed. F. Frenkel \& P. Sheppard (Advances in Geophysics, Vol. 6; New York: Academic Press), 161

[8] Dalena, S., Chuychai, P., Mace, R. L., Greco, A., Qin, G., \& Matthaeus, W. M. 2012, CoPhC, 183, 1974

[9] Ghilea, M. C., Ruffolo, D., Chuychai, P., Sonsrettee, W., Seripienlert, A., \& Matthaeus, W. H. 2011, ApJ, 741, 16

[10] Gleeson, L. J. 1969, P\&SS, 17, 31

[11] Jokipii, J. R. 1966, ApJ, 146, 480

[12] le Roux, J. A., \& Webb, G. M. 2007, ApJ, 667, 930

[13] Matthaeus, W. H., Goldstein, M. L., \& Roberts, D. A. 1990, JGR, 95, 20673

[14] Matthaeus, W. H., Qin, G., Bieber, J. W., \& Zank, G. 2003, ApJ, 590, L53

[15] Minnie, J., Bieber, J. W., Matthaeus, W. H., \& Burger, R. A. 2007, ApJ, 663, 1049

[16] Oughton, S., Dmitruk, P., \& Matthaeus, W. H. 2004, PhPl, 11, 2214

[17] Qin, G. 2007, ApJ, 656, 217

[18] Qin, G., \& Zhang, L.-H. 2014, ApJ, 787, 12

[19] Ruffolo, D., Chuychai, P., Wongpan, P., Minnie, J., Bieber, J. W., \& Matthaeus, W. H. 2008, ApJ, 686, 1231

[20] Ruffolo, D., \& Matthaeus, W. H. 2013, PhPl, 20, 012308

[21] Ruffolo, D., \& Matthaeus, W. H. 2015, ApJ, 806, 233

[22] Ruffolo, D., Pianpanit, T., Matthaeus, W. H., \& Chuychai, P. 2012, ApJ 747, L34

[23] Seripienlert, A., Ruffolo, D., Matthaeus, W. H., \& Chuychai, P. 2010, ApJ, 711, 980

[24] Shalchi, A. 2006, A\&A, 453, L43

[25] Shalchi, A. 2010, ApJ, 720, L127

[26] Shalchi, A. 2015, ApJ, 799, 232

[27] Shalchi, A., Bieber, J. W., Matthaeus, W. H., \& Qin, G. 2004, ApJ, 616, 617

[28] Shalchi, A., Bieber, J. W., Matthaeus, W. H., \& Schlickeiser, R. 2006, ApJ, 642, 230

[29] Shalchi, A., \& Hussein, M. 2014, ApJ, 794, 56

[30] Shebalin, J. V., Matthaeus, W. H., \& Montgomery, D. 1983, JPIPh, 29, 525

[31] Weygand, J. M., Matthaeus, W. H., Dasso, S., et al. 2009, JGR, 114, A07213 